PSICOLOGIA Vol. XI NN 1 y 21992

\title{
ADAPTACION DEL INVENTARIO DE ESTILOS DE APRENDIZAJE DE KOLB
}

\author{
Luis Miguel Escurra Mayaute*
}

El propósito de este trabajo es el de presentar el estudio de adaptación del Inventario de estilos de aprendizaje (IEA).

Se trabajó con 250 estudiantes Universitarios de ambos sexos pertenecientes a la especialidad de Psicología de U.N.M. de San Marcos y la P.U. Católica del Perú.

El inventario fue sometido a un estudio piloto que demostró la necesidad de cambiar el formato de aplicación. El análisis psicométrico incluyó el estudio de la Validez de Contenido y la validez de Construcción por medio del método interpruebas.

Los resultados obtenido permiten concluir que el inventario de estilos de aprendizaje en su versión Lima tiene validez y confiabilidad.
The purpose of this paper is to present the adaptation of Kolb Inventory of Cognitive Styles. The study was carried out with 250 psychology students of both genders from two universities of Lima.

A pilot study indicated the need to adapt the instrument. The psychometric analysis included a study content validity and construct validity through the iner-test method. The results showed that the inventory, in its Lima version, has validity ad reliability. 

En la actualidad, cuando se quiere adaptar una prueba Psicologica de una cultura a otra, se hace indispensable tomar en cuenta las diferencias que pueden existir entre ellas (Marín, 1986).

Dado que el Inventario de Estilos de Aprendizaje es la consecuencia natural de la aplicación de los conceptos teóricos de Kolb, para poder emplearlo en nuestro medio resulta indispensable demostrar la relación que existe entre la concepción teórica y su equivalencia cultural tanto en términos de la operacionalización del constructo,la equivalencia de los ítems y los puntajes escalares (Hui y Triandis, 1985; Marín, 1986).

El presente trabajo tiene por finalidad estudiar objetivamente los alcances y limitaciones de los fundamentos teóricos y metodológicos de la construcción de Pruebas Psicológicas aplicadas para la adaptación del Inventario de Estilos de Aprendizaje (IEA) de Kolb en una muestra de estudiantes de Psicología pertenecientes a dos Universidades de Lima Metropolitana.

\section{El Modelo de Aprendizaje Experiencial de Kolb}

Kolb, propone un modelo para examinar la forma cómo aprenden los sujetos a partir de la experiencia, y que toma en cuenta los aportes tanto de Jung, Lewin así como de Piaget (Kolb, 1979, 1981).

Kolb considera que el aprendizaje constituye un modelo cíclico, con 4 estados básicos y que incluyen las siguientes fases: a) Experiencia Concreta (EC) de una situación de aprendizaje, b) Observación Reflexiva (OR) de un fenómeno relevante, c) Conceptualización Abstracta (CA) acerca de lo que significa aquello que es observado y d) Experimentación Activa (EA) de las hipótesis planteadas.

A partir de la preferencia por una determinada fase de aprendizaje, Kolb (1979) definio los siguientes tipos de Aprendizaje: 


\section{Convergente}

Pone énfasis en la conceptualización abstracta y la experimentación activa. Estas personas se caracterizan por aplicar sus ideas para solucionar problemas.

Sus intereses se centran más en el mundo de las ideas que de las personas y tienden a especializarse en el campo tecnológico y científico.

\section{Divergente.}

Enfatiza la experiencia concreta y la observación reflexiva como formas de aprender, son personas que ticnen gran imaginación y son capaces de analizar las experiencias desde varios puntos de vista, sus intereses se centran en trabajar principalmente con las personas.

\section{Asimilador}

Prioriza la conceptualización abstracta y la observación reflexiva, se interesa por usar su razonamiento inductivo y construir teorras que permitan asimilar la observación a un marco de trabajo integrado, sus intereses se orientan por las matemáticas y otros campos de las ciencias básicas.

\section{Acomodador}

Se caracteriza por centrarse más en la experiencia concreta y la experimentación activa, se interesa más por hacer cosas y plantear soluciones a los problemas a partir de sus propias experiencias, sus intereses se orientan principalmente hacia el campo de los negocios.

\section{El Inventario Estilo de Aprendizaje de Kolb}

Este instrumento fué construido por D.A. KOLB en 1975 (Kolb, 1979) tomando como base conceptual su modelo experiencial. Evalúa la preferencia por un determinado estilo de aprendizaje, comparando los relativos predominios de una particular modalidad de aprender entre todas las posibles modalidades definidas por el modelo.

El inventario, siguiendo a Kerlinger $(1975,1979)$, es clasificado como una medida ipsativa. Su aplicación puede ser individual o grupal, siendo en algunos casos autoadministrado.

Está constituido por 36 palabras, de las cuales 24 están asociadas a cada una de las cuatro fases del ciclo de aprendizaje experiencial, las 12 palabras restantes son incluidas como elementos distractores para controlar la deseabilidad social, de ahf que no sean utilizadas para el cómputo final. 
Las 24 palabras evalúan las 4 etapas del aprendizaje experiencial, así como las siguientes dimensiones: a) Concreta - Abstracta, y b) Actividad - Reflexión.

En inventario consta de 9 grupos de 4 palabras cada uno, los sujetos deben responder ordenando jerárquicamente cada grupo de palabras, según el grado con el cual se percibe a la palabra que mejor caracteriza su estilo individual de aprender, asignando los puntajes que van de 1 a 4 (del menos al más característico).

El estudio inicial para la estandarización del instrumento, se realiz6 con una muestra de 287 sujetos, entre directivos y estudiantes de post-grado de administración de empresas de una universidad Norteamericana, con los cuales se obtuvieron los datos iniciales de la validez y confiabilidad del Inventario de Estilos de Aprendizaje (Leaming Style Inventory - LSI, Kolb 1979).

La validez del L.S.I., fue determinada de diferentes formas, en un principio Kolb (1979), estudió la validez de construcción en base a las modalidades Convergente y Discriminante, llegando a la conclusión de que la prueba tenía ambos tipos de validez, pues los resultados encontrados corroboraron las hipotesis planteadas.

Kolb (1984) inđica que Gypen, en 1980, evaluó la validez concurrente del LSI con la escala de evaluación de la orientación del aprendizaje hacia el trabajo, los resultados en este caso también permitieron concluir que el LSI presentaba validez concurrente.

Merrit y Marshall (1984) por su parte, evaluaron la validez de construcción del LSI según los supuestos del modelo de Kolb, utilizando para ello 2 versiones del instrumento. Utilizaron la forma ipsativa tradicional y otra forma de tipo normativo, donde cada palabra se podía calificar según el grado de importancia en un continuo de 4 puntos, los resultados permitieron apreciar que para el caso del LSI en su forma ipsativa, existen dos factores bipolares, los cuales correspondían a las 2 dimensiones básicas del aprendizaje experiencial; en tanto que el LSI en su forma normativa denotó existencia de 4 factores, cada uno correspondiente a cada fase del aprendizaje, con lo cual queda demostrada la validez de construcción del instrumento.

Con respecto a la Confiabilidad del LSI, Kolb originalmente evaluó la consistencia interna, por medio del método de la división por mitades (SpiltHalf), así como la estabilidad en el tiempo bajo la modalidad test-retest, los resultados permitieron concluir que el instrumento es confiable.

En otro estudio, Merrit y Marshall (1984), trabajando con dos versiones del LSI, evaluaron la consistencia interna de las pruebas a través del coeficiente Alfa de Cronbach. Para el caso de la versión ipsativa se encontró que los resultados oscilan entre 0.29 y 0.59 siendo su Alfa promedio igual a 0.46 , 
mientras que para la versión normativa los valores obtenidos van desde $\mathbf{0 . 5 2}$ a 0.74 siendo su promedio de $\mathbf{0 . 6 0}$.

Wilson (1986), trabajando con 130 alumnos de cursos de administración, aplico el LSI y estudió su confiabilidad tanto por el método de las mitades como por el test-retest; los resultados indicaron que la confiabilidad del LSI era moderada.

Las tablas normativas del LSI, fueron construidas en base a una muestra de 800 sujetos, 227 de los cuales eran gerentes en actividad y 573 graduados en administración de empresas.

Los puntajes directos de cada una de las 4 fases de aprendizaje, así como sus dimensiones respectivas fueron convertidas a rangos percentiles. Para la elaboración del perfil de estilo de aprendizaje se utiliza un nomograma circular de las cuatro fases, con círculos concéntricos que representan los percentiles $20,40,60,80$, y 100 .

El estilo de aprendizaje es determinado en base al nomograma de clasificación, construido en base a ejes coordenados que unen las dos dimensiones básicas del aprendizaje, los cuales se intersectan en sus puntajes promedios respectivos.

\section{Método}

Variables estudiadas

Fueron las siguientes:

a) Edad: Considerada como la edad cronológica del sujeto al momento de aplicarse la prueba, la cual puede ser entre los 20 y 35 ańos.

b) Sexo: Femenino o masculino

c) Universidad de Procedencia: El sujeto debe ser alumno regular de la universidad a la que pertenece (cursar como mínimo 12 créditos en el semestre en que se aplicó la prueba). Pueden pertenecer a la Universidad Nacional Mayor de San Marcos (UNMSM) o a la Pontificia Universidad Católica del Perú (PUC).

d) Estilos de Aprendizaje: Medidos a través de los puntajes del Inventario de Estilos de Aprendizaje, obtenidos para las áreas de: Experimentación Activa, Observación Reflexiva, Conceptualización Abstracta, Experiencia Concreta; asi como las dimensiones: Concreta-Abstracta, Actividad-Reflexión. 


\section{Muestra}

Se trabajó con 250 estudiantes de Psicología pertenecientes a la UNMSM (69.20\%) y la PUC (30.80\%), de ambos sexos (masculino, 38\% y femenino, $62 \%$ ), que han seguido 4 ciclos de estudios y con edades que fluctúan entre los 20 y 35 anos.

La muestra fue obtenida en base a un muestreo intencional de tipo no probabilístico y el tamano de la misma se obtuvo aplicando la fórmula de Cochran (1981).

\section{Estrategía de Adaptación}

La adaptación de la prueba se realizó siguiendo los siguientes pasos:

\section{Diseño del Instrumento}

Se procedió siguiendo a Brislin (1970) a someter a la prueba original en inglés, a un proceso de doble traducción contándose con el apoyo de dos personas bilingües, las cuales tradujeron la prueba del idioma inglés al español, para posteriormente traducir esta versión española al idioma inglés.

Al no encontrarse mayores diferencias en los resultados, se diseño la prueba inicial con las palabras que mejor denotaban la equivalencia lingüística (Marín, 1986).

Con este formato inicial se efectuó una pequeña prueba piloto, siendo aplicada a 10 sujetos, con la finalidad de evaluar la adecuación del formato.

Los sujetos a quienes se aplicó la prueba, indicaron de forma muy clara el tener dificultades para poder interpretar el significado de los ítemes (palabras), lo cual motivó que en muchos casos se emitiera una respuesta sin mayor consistencia, por lo que fue necesario realizar otra aplicación del instrumento en la cual se evaluó el significado que daban los sujetos a los ítemes del inventario.

\section{Evaluación de la Prueba Inicial}

Se aplicó a 30 sujetos una versión modificada del inventario, en la cual ellos debían tratar de indicar cuál era el significado de cada uno de los ítemes.

Para determinar la existencia de diferencias y puesto que los datos obtenidos podían ser clasificados de acuerdo a categorías, se utilizo siguiendo a Reuchlin (1980), el índice de diversidad para escalas nominales (H), así como su correspondiente docimacia estadística a través del índice J de Shanon (Zar, 1984). 
Los resultados encontrados, denotaron la necesidad de realizar algunos cambios para garantizar el buen funcionamiento del instrumento.

3. Construcción de la versión final del Inventario de Estilos de Aprendizaje

Fue necesario definir las palabras (ítemes) para lograr una mayor concordancia en los criterios usados para responder al inventario.

De esta forma se construyó la versión final, con la cual se realizó el trabajo en su integridad.

\section{Validez del Contenido}

Se estudió la validez de contenido, a través del método del criterio de jueces (Andreani, 1975; Aiken, 1980; Escurra, 1988).

Este proceso consistio en presentar a 10 especialistas, un formato del inventario así como las definiciones de las áreas y dimensiones del instrumento para que evaluaran el grado en que las palabras concordaban con las definiciones propuestas, así como el grado en que los ítemes pudieran estar midiendo el constructo estudiado.

Las opiniones fueron emitidas tanto de manera escrita como verbal, cuantificándose sus respuestas, en términos de acuerdo o desacuerdo con cada ítem del inventario.

Los resultados fueron evaluados cuantitativamente a través del coeficiente V de Aiken (Aiken, 1980, 1985; Escurra, 1988).

\section{Validez de Construcción}

Se trató de evaluar el constructo que sustentaba la prueba, obteniéndose la mayor cantidad posible de evidencias, de manera que se pudo evaluar la adecuación tanto de la prueba como de la teoría que la originaba, para ello se utilizó el método intraprueba, según Cronbach y Meehl (1971).

Este método consiste en estudiar la estructura interma de la prueba, efectuándose diversos análisis de las cualidades Psicométricas de la prueba. Para ello se estructuró una matriz de correlaciones de Pearson entre los ítemes, áreas y demensiones.

Los resultados fueron analizados teniendo como base la siguiente Red Nomológica, que equivale a un conjunto de hipótesis respecto a la prueba y que deben ser evaluadas. Las Hipótesis planteadas fueron, $\mathrm{H}_{1}$ : Los ítemes correlacionan positiva y significativamente con el total del área a la que pertenecen, $\mathrm{H}_{2}$ : Los ítemes de un área correlacionan negativa y significativamente con el 
total del área complementaria, con la que conforman una dimensión y $\mathrm{H}_{3}$ : Las áreas correlacionan de forma negativa y significativa con sus áreas complementarias.

Adicionalmente se realizó el Análisis Factorial, para verificar si las dimensiones propuestas por Kolb emergen en la base de datos recogidos. en este estudio.

\section{Confiabilidad}

Se estudio la confiabilidad a través đe los métodos que implican una sola aplicación del instrumento, debido a que es concordante con el marco teórico de Kolb.

La consistencia interna fue estudiada en base a las siguientes modalidades:

a) Homogeneidad. Que utiliza todos los ítemes en forma simultánea, para determinar la consistencia interna de la prueba (Brown, 1980: Klein, 1986). Se utilizaron los coeficientes Alfa de Cronbach y de Castaños.

b) Método de la División por Mitades (Par-Impar). Que consiste en dividir la prueba en dos partes iguales, en este caso, la división fue efectuada para cada una de las áreas del inventario.

Para la cuantificación de los datos, se computó la correlación simple de Pearson y la formula profética de Spearman Brown, así como los coeficientes de Guttman y Rulon.

\section{Baremos}

Las puntuaciones directas tanto por áreas como por dimensiones fueron transformadas a Rango Percentil, con la finalidad de poder contar con un sistema de medida que posibilite la comparación de los individuos. Adicionalmente a la presentación numérica de los puntajes, se diseño un nomograma, que permite expresar los resultados de forma gráfica.

\section{Procedimiento de Recolección de Datos}

La fase de recolección de datos se realizo entre los meses de Enero y Junio de 1990. Las aplicaciones se hicieron de forma colectiva y simultánea en la UNMSM y la PUC.

Se seleccionaron las aulas en las que se impartían los cursos de la carrera de Psicologfa, efectuándose las aplicaciones en forma grupal. Duranic esta fase, se tuvo el apoyo de 2 asistentes. 


\section{Resultados}

$\mathrm{El}$ análisis inicial fue efectuado en base al indice de la información $\mathrm{H}$ de los ítemes revisados, encontrándose en todas las áreas índices muy pequeños, indicando que las palabras presentan respuestas con muy diversos significados, por lo que tanto el $\mathbf{H}$ máximo como el índice $\mathrm{J}$ de Shanon alcanzan valores elevados, debiéndose por ello rechazar estos ítemes. Además notamos que sólo 3 palabras presentaban respuestas consistentes, por lo que se procedio a revisar la prueba, de modo que se optó por definir operacionalmente cada palabra con la finalidad de establecer de forma clara que aspectos eran evaluados por cada una de ellas y así tener un instrumento más adecuado para nuestra realidad. Una vez definidas cada una de las palabras, fueron sometidas a un proceso de validación con un grupo de jueces.

Los resultados obtenidos con el coeficiente $V$ de Aiken, nos indican que las palabras alcanzan coeficientes iguales o mayores que 0.80 obteniéndose una $\mathrm{p}$ asociada de .05 o menos, por lo que todos los ftemes son aceptados significativamente por los jueces, como muestras de conductas representativas de los estilos de aprendizaje y concluimos que el inventario tiene Validez de Contenido.

Al estudiar la Red Nomológica del instrumento, encontramos que las correlaciones corregidas de los ftemes con el puntaje total tanto de cada área como del área complementaria, nos indican que para cada dimensión los valores son estadísticamente significativos, tanto en términos positivos como negativos, validándose las hipótesis $\mathrm{H}_{1}$ y $\mathrm{H}_{2}$.

En el Cuadro $N^{q} 1$ observamos que existen correlaciones estadísticamente significativas entre las áreas y sus dimensiones correspondientes, EC y CA con CA-EC, así como entre EA y OR con EA-OR. También notamos que existen relaciones significativas y negativas entre EC y CA y entre EA y OR, resultados que confirman la validez de la hipótesis $\mathrm{H}_{3}$.

En conclusión, los resultados confirman la adecuación de la Red Nomológica, por el cual el instrumento presenta Validez de Construcción.

Prosiguiendo con el análisis de la Validez de Constructo, se efectuó el estudio de la composición factorial del instrumento, previamente se aplicó la prueba $\mathrm{X}_{2}$ de Steiger de bondad de ajuste a la matriz Identidad de los ftemes del IEA, obteniéndose un resultado de 16,509.48 con 276 grados de libertad y que alcanza una probabilidad asociada de .000 , lo que nos indica que existen diferencias significativas entre la matriz estudiada y la matriz identidad, confirmándose la posibilidad de realizar el análisis factorial y poder encontrar resultados significativos. 


\section{Cuadro $N^{2} 1$}

Matríz de Correlación entre áreas y Dimensiones del IEA

\begin{tabular}{llllll}
\hline & EC & OR & CA & EA & CA-EC \\
\hline OR & -0.129 & & & & \\
CA & $-0.579 *$ & -0.256 & & & \\
EA & $-0.442 *$ & $-0.664 *$ & -0.085 & & \\
CA-EC & $-0.853^{*}$ & 0.362 & $0.867 *$ & 0.244 & \\
EA-OR & -0.364 & $0.888 *$ & -0.037 & $0.880 *$ & $0.572 *$ \\
\hline
\end{tabular}

* Significativo $p<.05 \mathrm{n}=250$

Al efectuar el Análisis Factorial por el método de los componentes principales y aplicar el método de solución Varimax encontramos que, en primer lugar, dos factores permiten explicar el 52.48\% de la varianza total (Cuadro $N^{Q}$. 2), siendo un buen indicio de la composición factorial de los ítemes.

En segundo lugar, notamos que los factores tienen una estructura jerárquica especifica, ya que el mayor peso factorial corresponde a la dimensión CA-EC (Concreta-Abstracta) y el menor a la dimensión EA-OR (Actividad-Reflexión); y ambos presentan un índice de adecuación escalar mayor de 0.90 , por lo que según Kaiser podemos clasificarlos como sobresalientés, garantizándonos la precisión en la definición de las áreas en base a los ítemes.

La composición factorial encontrada en los ítemes confirma la. propuesta teorica ya que ambos factores saturan de forma tanto positiva como negativa determinados ítemes, y constituyen las 2 dimensiones básicas del modelo (Concreta-Abstracta y Experimentación-Observacion).

Cuando analizamos la significación estadística de los factores encontrados en los resultados del Análisis de traslapamiento y del Análisis paralelo, notamos que éstos nos indican que en ambos casos es factible aceptar como válidos los 2 factores; con lo cual se confirma la Validez teorica del IEA y su correspondencia con el modelo de Kolb, en lo que se refiere a los factores subyacentes a la composición de los ítemes.

El análisis de la confiabilidad realizados a través del estudio de la homogeneidad, nos indica que tanto las áreas como las dimensiones alcanzan coeficientes Alfa de Cronbach $(\alpha)$ que oscilan entre 0.67 y 0.87 y valores en el coeficiente de Castaños que van desde 0.75 hasta 0.89 (Cuadro $N^{Q} 3$ ), con lo que podemos concluir que el IEA es un instrumento que tiene confiabilidad.

Resulta necesario resaltar que entre el coeficiente Alfa de Cronbach y el de Castaños, existe una mínima discrepancia, pues el segundo tiende a dar 


\section{Cuadro $\mathbf{N}^{2} 2$}

Análisis Factorial Solución Varimax de los Itemes del IEA

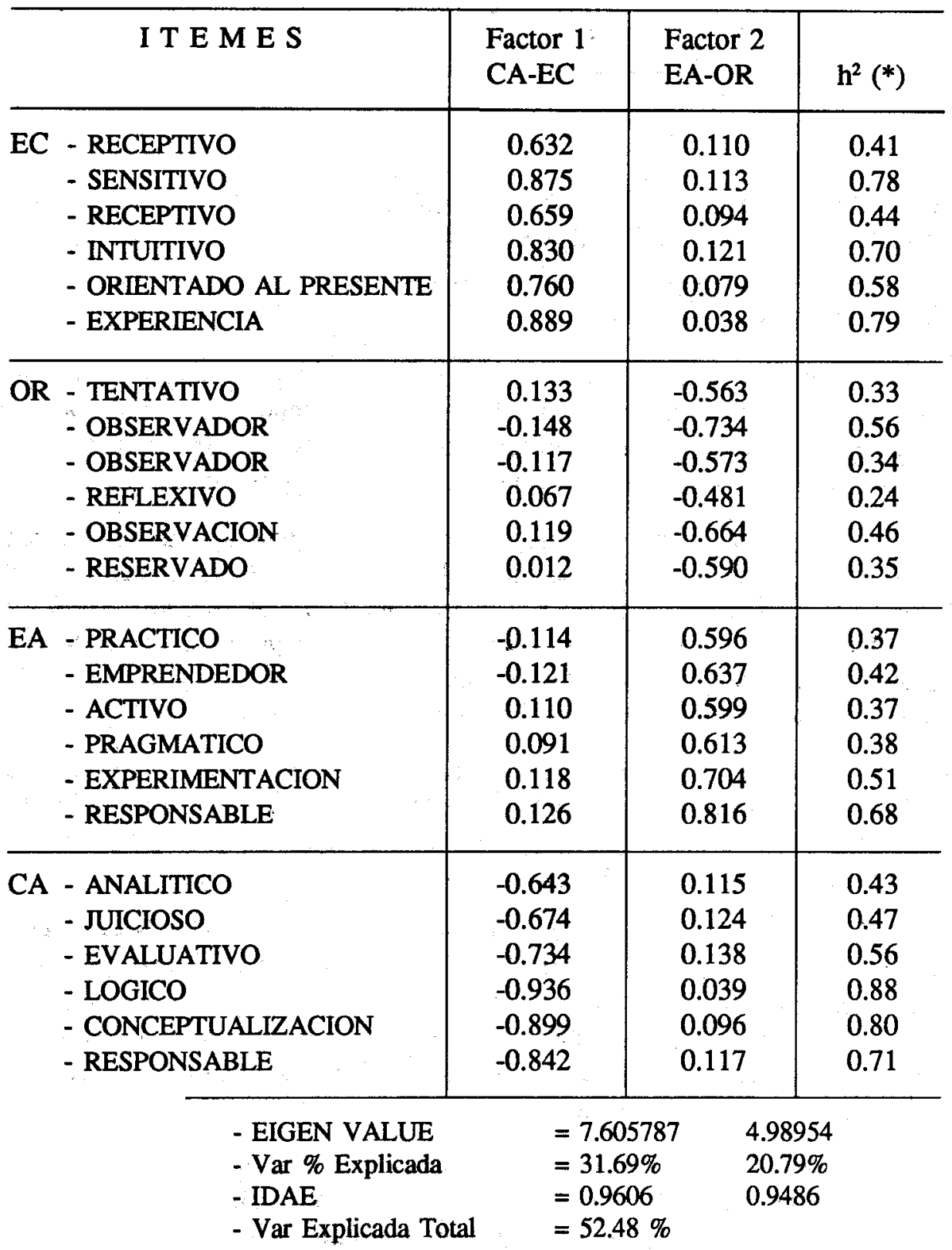

Estos resultados corroboran la Validez del instrumento. 
resultados ligeramente más elevados en todas las áreas y dimensiones estudiadas, esto puede deberse al algoritmo utilizado para el cómputo, ya que mientras el Alfa emplea la varianza de los ítemes, el de Castaños hace uso de las correlaciones ftem-test.

Entonces es factible utilizar cualquiera de estos dos coeficientes para computar la confiabilidad, sin modificar en mucho los resultados.

Otro resultado interesante estriba en que los promedios de las correlaciones para cada aspecto, son siempre menores que cualquiera de los 2 coeficientes antes nombrados. En tanto que un estudio más psicométrico, como es el caso del cuadrado de las correlaciones promedio, nos permite conocer en términos generales qué porcentaje del puntaje obtenido se debe a la puntuación verdadera del sujeto, encontrándose que los valores alcanzados son superiores a 0.79 , lo que nos permite determinar el grado con el que los sujetos al responder evidencian su verdadero estilo de aprendizaje.

\section{Cuadro $N^{2} 3$}

Coeficientes de Confiabilidad por Consistencia Interna y Homogeneidad

\begin{tabular}{l|c|c|c|c|c|c}
\hline & EC & OR & CA & EA & CA-EC & EA-OR \\
\hline$\alpha$ & 0.75 & 0.75 & 0.69 & 0.67 & 0.82 & 0.87 \\
CASTAÑOS & 0.77 & 0.77 & 0.73 & 0.70 & 0.85 & 0.89 \\
$r$ & 0.67 & 0.67 & 0.65 & 0.61 & 0.61 & 0.67 \\
$\mathbf{r}$ & 0.82 & 0.82 & 0.81 & 0.79 & 0.79 & 0.82 \\
\hline
\end{tabular}

En el estudio de la Confiabilidad a través de la consistencia interna por medio del método de la división por mitades, (Cuadro $N^{\circ} 4$ ) apreciamos que los resultados de los coeficientes tanto de Spearman-Brown, Guttman y Rulon, alcanzan valores que oscilan entre 0.74 y 0.72 , los cual nos indica que la prueba a través de este método también tiene confiabilidad y que los índices utilizados otorgan resultados equivalentes entre sí.

Es a partir de estos hallazgos que en lo referido a la confiabilidad, conclúmos que el instrumento es confiable en términos de su consistencia interna y homogeneidad, corroborándose lo postulado por Kolb.

Al analizar los resultados descriptivos (Cuadro $N^{0} 5$ ), notamos que los alumnos de la UNMSM obtienen el valor promedio más alto en el área de Conceptualización Abstracta y el valor promedio más bajo en la Experimen- 
Cuadro № 4

Coeficientes de Consistencia Interna de División por Mitades

\begin{tabular}{l|c|c|c|c|c|c}
\hline & EC & OR & CA & EA & CA-EC & EA-OR \\
\hline $\mathrm{r}$ & 0.66 & 0.68 & 0.66 & 0.64 & 0.80 & 0.87 \\
$\mathrm{r}$ & 0.79 & 0.81 & 0.80 & 0.78 & 0.86 & 0.93 \\
Guttman & 0.76 & 0.79 & 0.78 & 0.74 & 0.83 & 0.92 \\
Rulon & 0.76 & 0.79 & 0.78 & 0.74 & 0.83 & 0.92 \\
\hline
\end{tabular}

tación Activa; en tanto que los alumnos de la PUC puntúan más en Conceptualización Abstracta y menos en Observación Reflexiva.

Por otro lado, con respecto al sexo apreciamos que tanto los hombres como las mujeres alcanzan un promedio más alto en Conceptualización Abstracta y un promedio más bajo en Experimentación Activa, cabe notar que en términos globales al combinarse los puntajes y obtener los promedios generales, esta tendencia se mantiene.

\section{Cuadro № 5}

Análisis Descriptivos de las Variables Estudiadas

\begin{tabular}{|c|c|c|c|c|c|c|c|c|}
\hline & & & EC & OR & $\mathrm{CA}$ & EA & CA-EC & EA-OR \\
\hline \multirow{4}{*}{$\begin{array}{l}\mathrm{U} \\
\mathrm{N} \\
\mathrm{I} \\
\mathrm{V} \\
\mathrm{E} \\
\mathrm{R} \\
\mathrm{S} .\end{array}$} & UNMSM & $\mathrm{X}$ & 15.26 & 15.73 & 16.73 & 14.81 & 1.48 & -0.92 \\
\hline & \multirow{2}{*}{$\begin{array}{l}\mathrm{n}=173 \\
\text { PUC }\end{array}$} & DS & 3.10 & 3.40 & 3.26 & 3.23 & 5.51 & 5.88 \\
\hline & & $\mathrm{X}$ & 15.46 & 14.76 & 16.93 & 14.99 & 1.47 & 0.23 \\
\hline & $\mathrm{n}=77$ & DS & 3.04 & 3.10 & 3.17 & 3.20 & 5.27 & 5.55 \\
\hline \multirow{4}{*}{$\begin{array}{l}\mathbf{S} \\
\mathbf{E} \\
\mathbf{X} \\
\mathbf{O}\end{array}$} & \multirow{2}{*}{$\begin{array}{l}\text { MASC } \\
n=95\end{array}$} & $\mathbf{X}$ & 15.13 & 15.70 & 16.72 & 14.90 & 1.59 & -0.80 \\
\hline & & DS & 3.26 & 3.26 & 3.28 & 3.31 & 5.62 & 5.76 \\
\hline & \multirow{2}{*}{$\begin{array}{l}\text { FEM } \\
n=155\end{array}$} & $X$ & 15.48 & 15.13 & 16.87 & 14.86 & 1.39 & -0.27 \\
\hline & & DS & 3.49 & 3.35 & 3.18 & 3.15 & 5.77 & 5.80 \\
\hline \multirow{2}{*}{ TOTAL } & \multirow[b]{2}{*}{$\mathrm{N}=250$} & $\underline{X}$ & 15.33 & 15.37 & 16.81 & 14.88 & 1.47 & -0.49 \\
\hline & & DS & 3.09 & 3.33 & 3.23 & 3.22 & 5.43 & 5.79 \\
\hline
\end{tabular}

Con relación al análisis inferencial (Cuadro $N^{\circ} 6$ ), efectuado en base a la contrastación de los grupos a través de la prueba $\mathrm{Z}$ de diferencia de medias, encontramos que para el estudio según la procedencia. Universitaria, sólo en el 
caso de la observación reflexiva existen diferencias significativas, a favor de los alumnos de la UNMSM; en tanto que en los otros casos los resultados indican que no existen diferencias entre los dos grupos, y si las hay éstas son productos del azar.

Por otro lado, es digno de destacar que en la dimension (ExperimentaciónObservación, EA-OR) se aprecia una ligera tendencia a la diferenciación y ésta favorece en términos de observación a San Marcos y la experimentación a los de la PUC.

Cuando evaluamos las comparaciones según el sexo del sujeto, notamos que en ninguno de los casos existen diferencias significativas.

\section{Cuadro $\mathrm{N}^{0} 6$}

Prueba Z de Diferencias de Medias Por Universidad y Por Sexo

\begin{tabular}{l|c|c|c|c}
\hline \multirow{2}{*}{} & \multicolumn{2}{|c|}{ UNIVERSIDAD $^{1}$} & \multicolumn{2}{c}{ SEXO $^{2}$} \\
\cline { 2 - 5 } & $\mathrm{Z}$ & $\mathrm{P}$ & $\mathrm{Z}$ & $\mathrm{P}$ \\
\hline EC & -0.48 & .3156 & -0.80 & .2119 \\
OR & 2.22 & $.0132^{*}$ & 1.33 & .0918 \\
CA & -0.46 & .3238 & 0.36 & .3594 \\
EA & -0.41 & .3409 & -0.09 & .4641 \\
CA-EC & 0.14 & .4443 & 0.27 & .3936 \\
EA-OR & -1.48 & .0694 & -0.70 & .2420 \\
\hline
\end{tabular}

(*) Significativo si $\mathrm{p}<.05$

() No significativo

(1) Se resta UNMSM menos PUC

(2) Se resta Masculino menos Femenino.

Al determinar el tipo de aprendizaje que le corresponde a los sujetos estudiados utilizando los resultados obtenidos, encontramos que los alumnos de la UNMSM presentan mayormente un estilo de aprendizaje de tipo asimilador, mientras que en los de la PUC no se encuentra un estilo de aprendizaje definido, prevaleciendo el área de Experimentación Activa.

Para el caso de la clasificación por sexo, en las mujeres predomina un estilo de aprendizaje del tipo Acomodador y en los hombres del tipo Asimilador. 


\section{Conclusiones}

Los resultados obtenidos del presente estudio nos permiten inferir las siguientes conclusiones:

a) Con relación al Inventario de Estilos de Aprendizaje.

1. El Instrumento tiene Validez de Contenido por criterio de jueces y Validez de Construcción, por lo que el inventario de Kolb es válido en nuestro medio.

2. El Inventario tiene Confiabilidad por Homegeneidad y Consistencia Interna.

3. Los rangos percentiles de las áreas y dimensiones del I.E.A. son adecuados para caracterizar los estilos de aprendizaje.

4. Dados los cambios sustanciales introducidos al IEA, de ahora en adelante la versión realizada en el estudio será identificada como el Inventario de Estilos de Aprendizaje de Kolb versión E (IEA-E).

b) Con relación a la metodología para la construcción de pruebas psicológicas.

1. Resulta indispensable constatar que se mantenga el significado teórico de los ítemes de todas aquellas pruebas que se traduzcan de otros idiomas.

2. La Validez de Contenido de un instrumento psicológico debe ser evaluada tanto de forma cualitativa como cuantitativa para verificar la adecuación estadística de las evaluaciones hechas por los jueces.

3. La Validez de Construcción debe ser explicitada en una Red Nomológica que exprese la estructura del Constructo Psicológico estudiado, de manera que se expongan los aspectos que permitan evaluar objetivamente el constructo teórico que sustenta a las pruebas.

4. Al utilizar el método de la correlación Item-Test para realizar el análisis de ítemes, es indispensable que este resultado sea corregido eliminando dicho ítem de la puntuación total, con la finalidad de obtener los valores reales que reflejen la relación que existe entre ambos tipos de puntajes.

5. El uso del análisis Factorial, tanto para la investigación como para la construcción de pruebas Psicologicas, debe ser restringido sólo para aquellas matrices de variables que tengan altas correlaciones entre sí, pudiendo utilizarse la prueba de bondad de ajuste a la matriz identidad $\mathrm{X}^{2}$ de Steiger con ese fin.

6. La selección de los factores significativos producto del Análisis Factorial, debe ser realizada en base a los criterios estadísticos del Análisis de 
Traslapamiento y el Análisis Paralelo, por ser estos métodos más objetivos.

7. Los coeficientes Castaños y el Alfa de Cronbach proporcionan resultados equivalentes, por lo tanto, pueden ser empleados indistintamente para evaluar la Homogeneidad de las pruebas psicológicas.

8. Los coeficientes de Rulon, de Guttman y la fórmula profética de Spearman-Brown para computar la confiabilidad por mitades, dan resultados similares para los tests en que se miden rasgos psicologicos y en los cuales el estudio de la dificultad no es relevante, por lo cual en estos casos se puede hacer uso de cualquiera de dichos coeficientes.

c) Con relación a la comparación de los grupos de estudiantes examinados

1. En los sujetos del estudio encontramos que la Conceptualización Abstracta es el área más desarrollada y la Experimentación Activa la menos desarrollada, to que evidencia el impacto que tiene la modalidad que se asume en la enseñanza universitaria de la Psicología en nuestro medio.

2. La Orientación Reflexiva se encuentra más desarrollada en los alumnos de la UNMSM en comparación con los alumnos de la PUC.

3. La Experiencia Concreta, la Conceptualización Abstracta y la Experimentación Activa tienen niveles de desarrollo semejantes en las dos universidades estudiadas.

4. En los alumnos de la UNMSM predomina un estilo de aprendizaje del tipo asimilador.

5. En los alumnos de la PUC no se encuentra un estilo de aprendizaje definido, aunque prevalece en ellos el área de la Experimentación Activa.

6. Los hombres y las mujeres tienen valores similares en todas las áreas y dimensiones del IEA-E.

7. En las mujeres predomina un estilo de aprendizaje del tipo Acomodador.

8. En los hombres predomina un estilo de aprendizaje del tipo Asimilador.

\section{Referencias}

Aiken, L. (1980). Content Validity and Reliability of Single Items or Questionnaire, Educational and Psychological Measurement. № 40: 955-959. 
Aiken, L. (1985). Three Coeficients for Analyzing the Reliability and Validi of Ratings, Educational and Psychological Measurement. 45: 131-142.

Andreani, O. (1975). Aptitud Mental y Rendimiento Escolar, Barcelona, Editorial Herder.

Brislin, R. (1970). Translation for Cross-Cultural Research, Journal of CrossCultural Psychology Vol 1, No 3: 185-216.

Brown, F. (1980) Principios de la Medición en Psicología y Educación, México: Edit. El Manual Modemo.

Cronbach, L. (1972). Fundamentos de la Exploración Psicológica. Madrid: Biblioteca Nueva.

Cronbach, L. y Meehl (1971). La Validez del Constructo en los test Psicológicos, en Megargee E. Métrica de la Personalidad, Vol 9 México: Edit. Trillas.

Escurra N., L. M. (1988). Cuantificación de la Validez de Contenido por el Criterio de Jueces, Revista de Psicología, Año VI, Vol VI, Nros. 1 y 2 pp. 103-111.

Hui, H. y Triandis, H. (1985). Measurement in Cross-Cultural Psychology a Review and Comparison of Strategies, Journal of Cross-Cultural Psychology, Vol $16 \mathrm{~N}^{2} 2$ : 131-152.

Kerlinger, F. (1975). Investigación del Comportamiento Técnica y Metodología, Mexico; Nueva Edit. Interamericana S.A.

Kerlinger, F. (1979) Enfoque conceptual de la Investigación del comportamiento, México: Nueva Edit. Interamericana S.A.

Kline, P. (1986). A Handbook of Test Construction: Introduction to Psychometric Design, New York:Methuen And. Co. Ltd.

Kolb, D.A. (1979). Learning Style Inventory: Technical Manual, Boston: Mc Bier y Co.

Kolb, D.A. (1981). Experiential Learning Theory and the Learning Style Inventory a Reply to Freedman and Stumpf, Academy of Manegement Review, Vol 2: 289-296.

Kolb, D.A. (1984). Experiential Learning Experience as the Source of learning and Development, Englewood Cliffs, New Jersey: Prentice Hall.

Marin, G (1986). Consideraciones Metodológicas Básicās para conducir Investigaciones Psicológicas en América Latina, Acta Psiquiátrica y Psicológica de América Latina, pp. 183-192.

Merrit, S. y Marshall, J.C. (1984). Reliability and Construct Validity of Ipsative and Normative Forms of the LSI, Educational and Psychological Measurement, 44: 463-472.

Reuchlin, Maurice (1980). Compendio de Estadística, Método Conceptual, Madrid, Pablo del Río Editor.

Wilson, D. (1986). An Investigation of the properties of Kolb's Learning Style Inventory, Leadership And Organization Development Journal, Vol 7 № 3: 3-15.

Zar, J. (1974). Biostatistical Analysis, New Jersey: Prentice Hall. 